Poster (036)

Epithelial Ovarian Cancer including Borderline Tumor

https://doi.org/10.3802/jgo.2021.32.S1.036

\section{Visceral fat-to-muscle ratio is an independent factor to predict overall survival of the patients with epithelial ovarian, fallopian tube, or primary peritoneal cancer}

\section{Sooji Ham, Eun-Ju Lee," Jin Hwa Choi}

Chung-Ang University Hospital, Chung-Ang University School of Medicine, Seoul, Korea (ejlee@cau.ac.kr)

Objective: This study aimed to investigate the clinical significance of body composition in Korean women with epithelial ovarian, fallopian tube, or primary peritoneal cancer. Methods: A total 153 consecutive patients who underwent primary or interval cytoreductive surgery in Chung-Ang University Hospital, Seoul, Korea were retrospectively identified. The visceral and subcutaneous fat areas, skeletal muscle area and skeletal muscle index (SMI), were measured based on the pre-treatment computed tomography scans at the third lumbar vertebra. Fat-to-muscle ratios were calculated using total fat area (tFMR) or visceral fat area (vFMR). Sarcopenia was defined as a SMI $<38.7 \mathrm{~cm}^{2} / \mathrm{m}^{2}$ and tFMR and vFMR were divided into high or low groups based on the median value. Results: Univariate survival analysis showed that serous type, older age, higher International Federation of Gynecology and Obstetrics (FIGO) stage, neoadjuvant chemotherapy, suboptimality of debulking surgery, chemoresistance and high vFMR group were significantly associated with poor overall survival (OS), while sarcopenia and tFMR were not associated with OS. Multivariate survival analysis revealed that FIGO stage (hazard ratio $[\mathrm{HR}]=2.24$; $\mathrm{p}=0.025)$, chemo-responsiveness $(\mathrm{HR}=4.89 ; \mathrm{p}=0.006)$, and vFMR ( $H R=3.55 ; p=0.013$ ) were independent factors to predict OS. High vFMR group were significantly associated with old age, high body mass index, the present diabetes mellitus or hypertension, menopausal status, serous type, and neoadjuvant chemotherapy compared with those in low vFMR group.

Conclusion: In conclusion, the pretreatment vFMR could be independent prediction factor of the prognosis of Korean patients with epithelial ovarian, fallopian tube, or primary peritoneal cancer.

Poster (037)

Gynecologic Cancer Screening

https://doi.org/10.3802/jgo.2021.32.S1.037

\section{Clinicopathological characteristics of epithelial ovarian cancer in elderly patients}

\section{Suttikamon Sroiwatana, Nutthaporn Chandeying}

Faculty of Medicine Vajira Hospital, Navamindradhiraj University, Bangkok, Thailand (nutthaporn027@gmail.com)

Objective: To evaluate the clinicopathological characteristics and treatment of epithelial ovarian cancer (EOC) in elderly patients. Prognostic factors for survival were also studied. Methods: The study included EOC patients who aged 60 years or older and who received treatment in our institution between January 2010 to December 2019. Demographic data, clinicopathological characteristics, treatments and their outcomes were compared between the elderly (60-69 years old) and extremely elderly patients ( $>70$ years old). Prognostic factors for overall survival (OS) and progression-free survival (PFS) including age group of the patients were also studied. Results: A total of 144 patients were included in this study. Median ages of 109 patients in the elderly and 35 patients in the extremely elderly were 63 years (interquartile range [IQR], 61-65 years) and 76 years (IQR, 73-82 years) respectively. The extreme elderly patients had significantly higher frequency of co-morbidity particularly metabolic disease and less optimal primary surgery: $51.4 \%$ vs. $19.3 \%(\mathrm{p}<0.0001)$ and $28.6 \%$ vs. $47.7 \%(\mathrm{p}=0.047)$ respectively. Although worse prognostic features including poor performance status, advanced stage, non-endometrioid histology were demonstrated more frequently in the extreme elderly patients, the differences were not statistically significant. The overall response rates between the 2 groups were not significantly different $54.3 \%$ in the extreme elderly vs $62.4 \%$ in the elderly $(\mathrm{p}=0.121)$. With a median follow-up of 32.1 months, 3-year PFS and 3 -year OS were not significantly different between the 2 groups: $50.7 \%$ in elderly ( $95 \%$ confidence interval $[\mathrm{CI}]=40.09 \%-60.34 \%$ ) vs. $56.8 \%$ (95\% CI $=36.16 \%-73.01 \%)(\mathrm{p}=0.714)$ in the extremely elderly for PFS and $93.9 \%$ (95\% CI=86.86-97.23) vs. 86.2\% (95\% $\mathrm{CI}=66.77-94.64)(\mathrm{p}=0.201)$ for OS. Multivariate analysis reviewed only advanced stage and adjuvant therapy were independent prognostic factors for survivals.

Conclusion: Extremely elderly ( $>70$ years) EOC patients had more co-morbidity and less optimal primary surgery than the elderly patients (60-69 years). No significant differences of clinicopathological factors and survivals were found between the 2 age groups.

Poster (038)

Gynecologic Cancer Screening

https://doi.org/10.3802/jgo.2021.32.S1.038

Investigation of PARP inhibitor resistance
through the analysis of serially collected
circulating tumor DNA (ctDNA) in ovarian
cancer patients


Yeeun Shim, ${ }^{1}$ Yoo-Na Kim, ${ }^{2}$ Seung-Tae Lee, ${ }^{3,{ }^{*}}$ Jung-Yun Lee, ${ }^{2}$ Sung Hoon Kim, ${ }^{2}$ Jong Rak Choi ${ }^{3}$

'Department of Laboratory Medicine, Graduate School of Medical Science, Brain Korea 21 Project, Yonsei University College of Medicine, Seoul, Korea

${ }^{2}$ Department of Obstetrics and Gynecology, Yonsei University College of Medicine, Seoul, Korea

${ }^{3}$ Department of Laboratory Medicine, Yonsei University College of Medicine, Seoul, Korea (lee.st@yuhs.ac)

Objective: Poly (ADP-ribose) polymerase (PARP) inhibitors are used to treat patients with defective homologous recombination repair (HRR), such as deleterious mutations in BRCA1 or BRCA2. Reversion mutation and the subsequent restoration of HRR pathway has been suggested as main resistance mechanism. However, reversion mutations do not fully explain the resistance mechanism. In this study, we enrolled PARP inhibitor treated ovarian cancer patients with germline or somatic BRCA mutation $(\mathrm{n}=71)$ and analyzed circulating tumor DNA (ctDNA) obtained from relapsed patients after progression on PARP inhibitor $(\mathrm{n}=16)$.

Methods: From whole blood samples, ctDNA was extracted from plasma and germline DNA from buffy coat. Extracted DNA was target enriched with panel targeting 531 cancerrelated genes, and the resultant library was sequenced using the Illumina Novaseq 6000 sequencing system. The sequencing data was analyzed using our custom analysis pipeline.

Results: Among 16 relapsed patients, 11 patients had both before PARP inhibitor treatment and relapsed samples, 5 patients had only relapsed sample. Every patient had tissue NGS data. Analysis of ctDNA samples showed ctDNA-specific variants. BRCA reversion was found in two patients (12.5\%). Analysis of patient-matched paired samples before and after progression on PARP inhibitor showed an increase in intratumoral heterogeneity in post-progression samples. New variants in genes that are linked to DNA repair pathway were found to be associated with increase tumor mutation burden. Conclusion: In this study, we demonstrated profiling of ctDNA, a less invasive assay, can efficiently offer information on tumor heterogeneity and predict PARP inhibitor resistance.

Poster (039)

Rare Tumors \& Metastatic Tumors

https://doi.org/10.3802/jgo.2021.32.S1.039

\section{Mature cystic teratoma of the ovary with carcinoid component: a case report}

Dolly Lubis, "Andi Darma Putra, Hartono Tjahjadi

Faculty of Medicine of Indonesian University, Dr. Cipto Mangunkusumo Hospital, Jakarta, Indonesia (doelhappy@gmail.com)
Objective: Learnig about clinical manifestation and diagnosis of mature teratoma cases with malignant transformation. Methods: A 36 years old woman, P2A0, was referred from a primary hospital because of post-operative pathological examination revealed a malignancy. Earlier, she underwent bilateral salpingo-oophorectomy (BSO) at a primary hospital for indication benign ovarian cyst. After BSO procedure, postoperative pathology result revealed: In both ovary, tumor tissue composed of ectoderm, mesoderm, and endoderm components with tumor cells nucleated round/oval, vesicular, relatively uniform, fine-grained chromatin resembling "salt and pepper, partially solid." Immunohistochemistry result training pattern was consistent with carcinoid tumors.

Results: In conclusion, histological according to mature ovarian teratoma with carcinoid.

Conclusion: Malignant transformation of benign ovarian tumors, including mature teratoma, is very rare. In addition, the clinical findings and pre-operative examinations did not help a lot to establish the diagnosis. Often the diagnosis was discovered unexpectedly in post-operative specimens such as happened in this case.

Poster (040)

Epithelial Ovarian Cancer including Borderline Tumor

https://doi.org/10.3802/jgo.2021.32.S1.040

\section{Single-arm, phase II study of niraparib and bevacizumab maintenance in platinum- sensitive, recurrent ovarian cancer previously treated with a PARP-inhibitor: KGOG3056/ NIRVANA-R}

\author{
Junsik Park, ${ }^{1}$ Myong Cheol Lim, ${ }^{2}$ Jae-Kwan Lee, ${ }^{3}$ Dae Hoon Jeong, \\ Se Ik Kim, ${ }^{5}$ Min Chul Choi, ${ }^{6}$ Byoung Gie Kim, ${ }^{7}$ Jung-Yun Lee, ${ }^{1, *}$ \\ 'Yonsei University College of Medicine, Seoul, Korea (jungyunlee@yuhs.ac) \\ ${ }^{2}$ National Cancer Center, Goyang, Korea \\ ${ }^{3}$ Korea University, Seoul, Korea \\ ${ }^{4}$ Inje University, Busan, Korea \\ ${ }^{5}$ Seoul National University College of Medicine, Seoul, Korea \\ ${ }^{6} \mathrm{CHA}$ Bundang Medical Center, Seongnam, Korea \\ ${ }^{7}$ Sungkyunkwan University School of Medicine, Suwon, Korea
}

Background: Given the expanding clinical use of poly(ADPribose) polymerase inhibitors (PARPis), there is a significant need for optimal strategies with which to treat patients whose cancer progresses while using a PARPi. However, the treatment consensus after PARPi has not been established. PARPi and anti-angiogenic agent combination therapy is a promising option to treat relapsed patients exposed to a PARPi. The synergistic efficacy of dual maintenance therapy 